\title{
The tumorigenic role of DSPP and its potential regulation of the unfolded protein response and ER stress in oral cancer cells
}

\author{
IOANNIS GKOUVERIS ${ }^{1}$, NIKOLAOS G. NIKITAKIS ${ }^{2}$, JAYA ASEERVATHAM ${ }^{3}$ and KALU U.E. OGBUREKE ${ }^{3}$ \\ ${ }^{1}$ Division of Diagnostic and Surgical Sciences, UCLA School of Dentistry, Los Angeles, CA 90095, USA; \\ ${ }^{2}$ Department of Oral Medicine and Pathology, School of Dentistry, National and Kapodistrian University of Athens, \\ Athens 11527, Greece; ${ }^{3}$ Department of Diagnostic and Biomedical Sciences, School of Dentistry, \\ University of Texas Health Science Center at Houston, Houston, TX 77054, USA
}

Received January 16, 2018; Accepted May 14, 2018

DOI: $10.3892 /$ ijo.2018.4484

\begin{abstract}
Dentin sialophosphoprotein(DSPP) is upregulated in various human cancers, including head and neck squamous cell carcinoma. Cancer cells are commonly found under constant endoplasmic reticulum (ER) stress and exhibit increased levels of misfolded proteins, due to gene mutations and a stressful microenvironment. The present study examined the effects of DSPP silencing on the regulation of ER stress and the unfolded protein response (UPR) in oral cancer cells. A recently established stable DSPP short hairpin (sh)RNA-silenced OSC2 oral cancer cell line was used. The mRNA expression levels of ER stress-associated proteins, including $78 \mathrm{kDa}$ glucose-regulated protein (GRP78), sarcoplasmic/endoplasmic reticulum calcium ATPase 2b (SERCA2b), inositol 1,4,5-trisphosphate receptor (IP3r), protein kinase R-like endoplasmic reticulum kinase (PERK), serine/threonine-protein kinase/endoribonuclease IRE1 (IRE1), activating transcription factor 6 (ATF6) and matrix metalloproteinase 20 (MMP20), were assessed by reverse transcription-quantitative polymerase chain reaction. The expression levels of apoptosis-related [B-cell lymphoma 2 $(\mathrm{Bcl} 2), \mathrm{Bcl} 2$-associated $\mathrm{X}$ protein (Bax) and cytochrome $c$ ] and cell proliferation-related [proliferating cell nuclear antigen (PCNA)] proteins were analyzed by western blotting. Cell viability, apoptosis and migration were monitored by MTT assay, Annexin V-fluorescein isothiocyanate flow cytometry and wound-healing assay, respectively. In transiently transfected puromycin-free OSC2 cells, DSPP silencing markedly downregulated the mRNA expression levels of major ER stress regulators, including GRP78, SERCA2b, PERK, IRE1 and ATF6, as well as MMP20. DSPP silencing also resulted
\end{abstract}

Correspondence to: Professor Kalu U.E. Ogbureke, Department of Diagnostic and Biomedical Sciences, School of Dentistry, University of Texas Health Science Center at Houston, 7500 Cambridge Street, Houston, TX 77054, USA

E-mail: kalu.ogbureke@uth.tmc.edu

Key words: dentin sialophosphoprotein, oral squamous cell carcinoma, endoplasmic reticulum stress, regulation, homeostasis in decreased cell viability and migration, and enhanced apoptosis. Furthermore, PCNA and Bcl2 levels were decreased, whereas Bax and cytochrome $c$ protein levels were increased in DSPP-silenced OSC2 cells. Sustained puromycin treatment partially counteracted the effects of DSPP silencing on the mRNA expression levels of ER stress-related proteins and MMP20, and on the migratory capacity of OSC2 cells. However, following puromycin treatment of DSPP-silenced cells, cell viability was further reduced and apoptosis was enhanced. In conclusion, these data provide evidence to suggest that DSPP may be involved in ER stress mechanisms in oral squamous cell carcinoma, since its downregulation in OSC2 cells led to significant alterations in the levels of major ER stress-associated proteins, and subsequent collapse of the UPR system.

\section{Introduction}

Dentin sialophosphoprotein (DSPP) is a member of the small integrin-binding ligand N-linked glycoprotein family, which activates kinases and transcription factors, and controls signal transduction pathways that regulate cell adhesion, migration and survival (1). DSPP, the expression of which was previously thought to be limited to mineralizing tissues such as dentin and bone, is now known to be expressed in metabolically active ductal epithelial tissues, including normal salivary glands, nephrons and eccrine sweat glands (2-4). The DSPP gene encodes two main noncollagenous dentin proteins: Dentin sialoprotein (DSP) and dentin phosphoprotein (DPP), as well as the much smaller dentin glycoprotein (DGP) (5). DSP occupies the N-terminal domain and presents a Cys205 (the only cysteine in DSPP) domain (6,7). C-terminal DPP possesses 165 Asp-Ser-Ser sequence motifs, has a low isoelectric point of 2.84 and exhibits high calcium affinity $(7,8)$. The short DGP fragment is sandwiched between the N-terminal DSP and the C-terminal DPP. It is difficult to isolate the full-length DSPP protein because soon after its translation, matrix metalloproteinase (MMP)20 and MMP2 mediate the proteolytic fragmentation of DSPP into DSP, DPP and DGP (5). The role of DGP remains unclear, whereas the functional roles of DSP include initiation of dentin mineralization and DPP promotes dentin maturation (6). 
Within the past decade, the expression of DSPP has been detected in numerous human epithelial cancers, including breast, lung, colon, prostate and oral cancer (9-12). In oral squamous cell carcinoma (OSCC), DSPP is significantly upregulated in poorly differentiated lesions (12). Recently, we reported the coexpression and potential interactions of MMP20 and DSPP in human OSCC tissues and cell lines (13). Furthermore, the report established the specific cognate partnering of MMP20 and DSPP, which also is present in metabolically active ductal epithelial systems, such as the salivary gland and nephron (13). In particular, the DSP portion of DSPP strongly interacts with MMP20 promoter proximal elements (13).

Endoplasmic reticulum (ER) is a major store of intracellular calcium, which is central to regulating cellular calcium homeostasis and various calcium signaling pathways (14). ER serves a key role in proper protein folding and targeting during protein synthesis; unfolded proteins entering the ER are chemically modified in an oxidizing and calcium-rich environment (15). ER-regulated calcium homeostasis is mediated by various calcium proteins, the functions of which are modulated by several factors, including redox mechanisms $(16,17)$. These calcium-related proteins function as influx pumps, which transfer calcium from the cytosol to the lumen, as luminal storing buffers, and as controlled-release calcium channels to the cytosol (16). ER homeostasis is a dynamic process that often exhibits sensitivity to minor environmental alterations (18); therefore, changes in redox state, ischemic state, nutrient status and $\mathrm{Ca}^{2+}$ levels, high protein synthesis rate and inflammation may disrupt proper ER function, thus resulting in the accumulation of unfolded or misfolded proteins $(18,19)$. This outcome is referred to as ER stress, which in turn triggers numerous molecular signaling pathways aimed at preventing intraluminal accumulation and/or secretion of unfolded proteins, also known as the unfolded protein response (UPR), by promoting the degradation of misfolded proteins through the ER-associated protein degradation pathway $(20,21)$.

Disturbances in ER homeostasis are detected by three ER transmembrane proteins-sensors: Protein kinase R-like endoplasmic reticulum kinase (PERK), activating transcription factor 6 (ATF6a and b), and serine/threonine-protein kinase/ endoribonuclease IRE1 (IRE1). Initiation of the UPR and activation of sensor proteins and related downstream molecules reduce the duration of stress, re-establishe normal ER function and promote survival. However, depending on stress severity, the UPR may trigger apoptosis and cell death $(16,18)$. In addition, $78 \mathrm{kDa}$ glucose-regulated protein (GRP78), also known as binding immunoglobulin protein, had been proposed as the master UPR chaperone that negatively regulates PERK, ATF6 and IRE1 activity $(15,22)$.

The accumulation of unfolded proteins releases PERK, ATF6 and IRE1 from the strong GRP78 engagement $(15,22)$. The tumor microenvironment is characterized by a lack of nutrients, low $\mathrm{pH}$, hypoxia and oxidative stress $(23,24)$. Consequently, changes in ER stress and UPR serve a critical role in the ability of cancer cells to survive in this demanding environment and prevent ER stress-induced apoptosis $(25,26)$. Previous studies have reported that GRP78 cysteine oxidation enhances cell survival during stress (27), and that GRP78, PERK, IRE1 and ATF6 overexpression in cancer is correlated with cell proliferation and survival signaling (28-31). Similarly, sarcoplasmic/endoplasmic reticulum calcium ATPase (SERCA) has been proposed as a potential target for cancer treatment (15).

The present study aimed to investigate the potential association of DSPP with the ER homeostatic mechanism, the possible inter-regulatory effects in OSCC biology, and the molecular pathways implicated.

\section{Materials and methods}

Stable DSPP-silenced OSC2 cell lines. Our laboratory recently established stable lentiviral-mediated DSPP-, MMP20- and combined DSPP + MMP20-silenced OSC2 cells; OSC2 cells infected with scrambled short hairpin (sh)RNA (SCR) were used as controls (13). shRNA Plasmid A (cat. no. sc-108060), which was used as a negative control, was obtained from Santa Cruz Biotechnology, Inc. (Dallas, TX, USA). The stably silenced (75\% silencing) phenotype of these cell lines was routinely verified by western blotting (data not shown). Subsequently, cells were cultured as a monolayer in Dulbecco's modified Eagle's medium (DMEM)/F12 (Gibco; Thermo Fisher Scientific, Inc., Waltham, MA, USA) supplemented with $10 \%$ fetal bovine serum (FBS; Gibco; Thermo Fisher Scientific, Inc.), $1 \%$ penicillin/streptomycin and $500 \mathrm{ng} / \mathrm{ml}$ hydrocortisone (Sigma Aldrich; Merck KGaA, Darmstadt, Germany) at $37^{\circ} \mathrm{C}$ in the presence of $5 \% \mathrm{CO}_{2}$ humidified air. Untreated DSPP-silenced stable cells were incubated in media devoid of puromycin for 4 days prior to experimentation, whereas treated DSPP-silenced stable cells were continuously treated with $3 \mu \mathrm{g} / \mathrm{ml}$ puromycin (cat. no. sc-108071; Santa Cruz Biotechnology, Inc.). Omission of puromycin in the untreated DSPP-silenced group did not affect cell stability.

Reverse transcription-quantitative polymerase chain reaction (RT-qPCR) analysis. To evaluate the effects of DSPP silencing on the mRNA expression levels of proteins associated with ER stress and the UPR, GRP78, SERCA2b, inositol 1,4,5-trisphosphate receptor (IP3r), PERK, IRE1 and ATF6 expression was detected by RT-qPCR analysis. RT-qPCR was conducted on total RNA extracted from DSPP-silenced and SCR OSC2 cells according to established protocols. Briefly, total RNA was extracted from cells using TRIzol ${ }^{\circledR}$ reagent (cat. no. 15596-026; Invitrogen; Thermo Fisher Scientific, Inc.), according to a standardized protocol, and the concentration of each sample was measured. qSTAR (Origene Technologies, Inc., Rockville, MD, USA) qPCR primer pairs against human genes were used in the present study; the primer sequences were as follows (5'-3'): DSPP forward, CAACCATAGAGAAAGCAAACGCG and reverse, TTTCTGTTGCCACTGCTGGGAC; MMP20 forward, GACCAGACCACAATGAACGT and reverse, GTCC ACTTCTCAGGATTGTC; PERK forward, ATCCCCCATG GAACGACCTG and reverse, ACCCGCCAGGGACAAAA ATG; ATF6 forward, TTGGCATTTATAATACTGAACTAT GGA and reverse, TTTGATTTGCAGGGCTCAC; SERCA2b forward, TCATCTTCCAGATCACACCGC and reverse, GTC AAGACCAGAACATATC; IP3r forward, GGTTTCATTTG CAAGTTAATAAAG and reverse, AATGCTTTCATGGAA CACTCGGTC; IRE1 forward, CGGGAATTCGGCCGAGTC CTCGCCATG and reverse, CAAGCGGCCGCCTTTCCCA ACTATCACCACGCT; GRP78 forward, TGTTCAACCAATT ATCAGCAAACTC and reverse, TTCTGCTGTATCCTCTT 
CACCAGT; and $\beta$-actin forward, GTCTCCTCTGACTTCAA CAGCG and reverse, ACCACCCTGTTGCTGTAGCCAA. Briefly, total RNA $(1 \mu \mathrm{g})$ was reverse transcribed using iScript $^{\mathrm{TM}}$ RT Supermix (cat. no. 1708841; Bio-Rad Laboratories, Inc., Hercules, CA, USA), according to the manufacturer's protocol. RT-qPCR was conducted using synthesized cDNA on a qPCR machine using iTaq ${ }^{\mathrm{TM}}$ Universal SYBR ${ }^{\circledR}$ Green Supermix (cat, no. 1725124; Bio-Rad Laboratories, Inc.). PCR thermocycling was as follows: $94^{\circ} \mathrm{C}$ for $5 \mathrm{~min}$, followed by 40 cycles at $94^{\circ} \mathrm{C}$ for $30 \mathrm{sec}, 60^{\circ} \mathrm{C}$ for $20 \mathrm{sec}$ and $72^{\circ} \mathrm{C}$ for $40 \mathrm{sec}$, followed by a final extension step at $72^{\circ} \mathrm{C}$ for $5 \mathrm{~min}$. A standard curve was generated from three serial dilutions of cDNA. Samples, including negative controls, were analyzed in triplicate, and PCR products were verified using dissociation curve analysis. mRNA expression levels were normalized to $\beta$-actin and were analyzed using Bio-Rad CFX Manager ${ }^{\mathrm{TM}}$ software (Version Number 3.0; Bio-Rad Laboratories, Inc.). The $\log 2$-fold change between the SCR and experimental treated samples was calculated using the $2^{-\Delta \Delta \mathrm{Cq}}$ method (32).

Western blot analysis. Cells were washed twice with ice-cold PBS, and were then lysed with radioimmunoprecipation assay buffer [cat. no. 156034; Abcam, Cambridge, MA, USA; $50 \mathrm{mM}$ Tris (pH 7.4), $150 \mathrm{mM} \mathrm{NaCl}, 1 \%$ Triton X-100, $1 \%$ deoxycholic acid sodium salt, $0.1 \%$ sodium dodecyl sulfate, $100 \mathrm{mg} / \mathrm{ml}$ phenylmethylsulfonyl fluoride, $1 \mathrm{mg} / \mathrm{ml}$ aprotinin, $1 \mathrm{mM}$ dichlorodiphenyltrichlo-roethane and $1 \mathrm{mM}$ sodium orthovanadate] for $10 \mathrm{~min}$ at $4^{\circ} \mathrm{C}$. The wells were scraped, and the recovered cell products were centrifuged (Sorvall ${ }^{\mathrm{TM}} \mathrm{CC} 40$; Thermo Fisher Scientific, Inc.) at 40,000 x g for $15 \mathrm{~min}$ at $4^{\circ} \mathrm{C}$. The concentration of the recovered proteins was measured using the Bio-Rad Protein Assay kit (Bio-Rad Laboratories, Inc.), according to the manufacturer's protocol. Equal amount of proteins (30-50 $\mu \mathrm{g}$, depending on the particular protein) were separated by SDS-PAGE (10\% separation gel and 5\% spacer gel) and were electrotransferred to polyvinylidene difluoride membranes (Bio-Rad Laboratories, Inc.). Membranes were then placed in blocking solution for $1 \mathrm{~h}$ at room temperature, after which they were probed with the following primary antibodies overnight at $4^{\circ} \mathrm{C}$ : Mouse monoclonal B-cell lymphoma 2 (Bcl2; cat. no. sc-7382; 1:250), mouse monoclonal Bcl2-associated X protein (Bax; cat. no. sc-7480; 1:200), rabbit polyclonal cytochrome $c$ (cat. no. sc-7159; 1:200) and rabbit polyclonal proliferating cell nuclear antigen (PCNA; cat. no. sc-7907; 1:200) (all from Santa Cruz Biotechnology, Inc.). The film was then washed thoroughly and incubated with goat polyclonal anti-rabbit immunoglobulin $\mathrm{G}(\mathrm{IgG})$ horseradish peroxidaseconjugated secondary antibody (cat. no. sc-2030; 1:3,000; Santa Cruz Biotechnology, Inc.) or anti-mouse IgG antibody (cat. no. sc-2031; 1:3,000; Santa Cruz Biotechnology, Inc.) with agitation at room temperature $\left(25^{\circ} \mathrm{C}\right)$ for $1 \mathrm{~h}$. $\beta$-actin $(1: 2,000)$ was used as a loading control (cat. no. sc-47778; Santa Cruz Biotechnology, Inc.). Proteins were visualized using an enhanced chemiluminescence (ECL) system (Pierce ${ }^{\mathrm{TM}}$ ECL; Thermo Fisher Scientific, Inc) and band intensity was semiquantified using ImageJ software1.48 (https://imagej.nih. gov/ij/; National Institutes of Health, Bethesda, MD, USA).

MTT assay. Cell viability was assessed by detecting the conversion of MTT to formazan, which is induced by mitochondrial oxidation. Cell cultures $\left(5 \times 10^{3}\right.$ cells/well), including SCR or DSPP-silenced (puromycin-treated and puromycin-free) cells, were incubated in the presence of $0.5 \mathrm{mg} / \mathrm{ml}$ MTT for $3 \mathrm{~h}$ at $37^{\circ} \mathrm{C}$ in 96 -well plates. Water insoluble, purple formazan crystals were formed, indicating the presence of viable cells. Crystals were then dissolved in dimethyl sulfoxide and optical density (OD) values of the solutions were measured using a spectrophotometer at a wavelength of $570 \mathrm{~nm}$. Assays were performed in triplicate and data are presented as the means of OD values \pm standard error of the mean.

Apoptosis analyses by flow cytometry. For apoptosis analyses, Annexin V/propidium iodide (PI) staining of SCR and DSPP-silenced (puromycin-treated and puromycin-free) cells was conducted. Briefly, cells were washed with $1 \mathrm{X}$ PBS and resuspended at $10^{6}$ cells $/ \mathrm{ml}$ in Annexin V-binding buffer prior to aliquoting the suspension into $100 \mu \mathrm{l} /$ tube fractions. Subsequently, $5 \mu \mathrm{l}$ Annexin V-fluorescein isothiocyanate (FITC) and $10 \mu 1$ PI buffer were added to each tube and incubated in the dark for $15 \mathrm{~min}$ at room temperature. Finally, $400 \mu 11 \mathrm{X}$ Annexin V-binding buffer was added to each tube and flow cytometric analysis was performed within $1 \mathrm{~h}$. Samples were analyzed on a FACSCalibur flow cytometer (BD Biosciences, Franklin Lakes, NJ, USA). Gates in the right angle scatter versus forward scatter diagrams were used to exclude debris. At least 100,000 events were recorded prior to analysis. All flow cytometric data were analyzed using BD Cell Quest Pro software (Version 5.0; BD Biosciences).

Scratch wound-healing assay. Cells were cultured to $90 \%$ confluence in $35-\mathrm{mm}$ dishes and scratched with a sterile 200- $\mu 1$ pipette tip. The border of the denuded area was immediately marked with a fine line, and cells were incubated with DMEM/F12 containing 10\% FBS with or without puromycin at $37^{\circ} \mathrm{C}$. Cultures were photographed after $24 \mathrm{~h}$ using an inverted phase contrast microscope (Olympus Corporation, Tokyo, Japan). The assay was performed in triplicate.

Statistical analyses. Statistical analysis was performed using SPSS version 21 (IBM Corp., Armonk, NY, USA). Paired groups were compared using Student's t-test, whereas one-way analysis of variance was applied to compare multiple groups, followed by post-hoc pairwise comparisons with the application of Dunn's test. All experiments were performed in triplicate. $\mathrm{P}<0.05$ was considered to indicate a statistically significant difference.

\section{Results}

DSPP silencing downregulates critical ER stress and $U P R$-related protein expression in OSC2 cells. As shown in Fig. 1A, DSPP silencing in OSC2 cells resulted in a significant reduction in the mRNA expression levels of GRP78, SERCA2b, PERK and ATF6 ( $\mathrm{P}<0.05)$, whereas IRE1 and IP3r mRNA expression was not significantly altered compared with the SCR group. Conversely, DSPP-silenced OSC2 cells treated with puromycin exhibited reduced GRP78, SERCA2b, IP3r, PERK, IRE1 and ATF6 mRNA levels compared with in the puromycin-treated SCR group. However, decreases in mRNA expression between puromycin-treated DSPP-silenced 
A

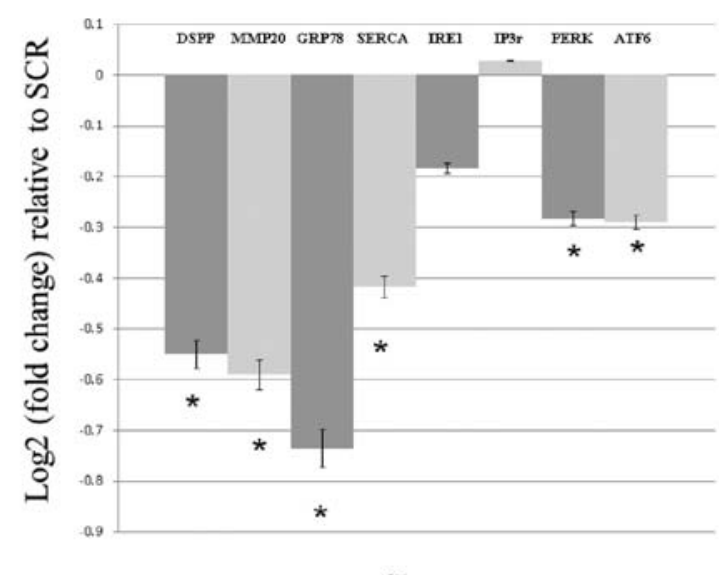

B

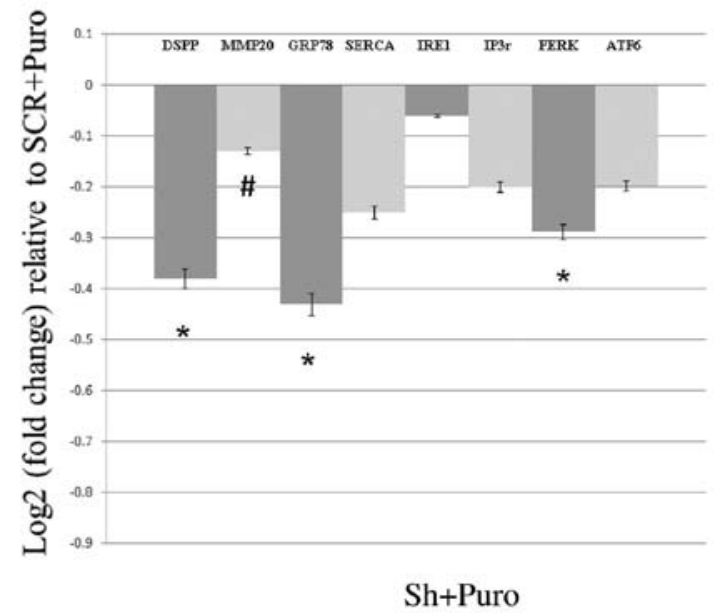

Figure 1. Effects of DSPP silencing on the mRNA expression levels of proteins associated with endoplasmic reticulum stress and the unfolded protein response in oral squamous cell carcinoma cells, as assessed by reverse transcription-quantitative polymerase chain reaction analyses. (A) DSPP mRNA expression exhibited a $0.55 \log 2$-fold change decrease in Sh compared with in SCR cells. mRNA expression levels of GRP78, SERCA2b, PERK and ATF6, as well as those of MMP20, were significantly reduced, whereas IRE1 and IP3r expression was not significantly altered compared with in SCR cells. (B) Puromycin treatment decreased DSPP silencing; the reduction in DSPP mRNA expression (38\%) was less pronounced compared with in DSPP-silenced puromycin-free cells (55\%). MMP20 mRNA expression was only minimally decreased in DSPP-silenced puromycin-treated cells, and puromycin-treated DSPP-silenced cells exhibited significantly $(" \mathrm{P}<0.05)$ increased MMP20 mRNA expression compared with in puromycin-free DSPP-silenced cells. Results are expressed as $\log 2$-fold changes relative to the expression levels in SCR cells. Data are presented as the means \pm standard error of the mean; each experiment was performed in triplicate. ${ }^{*} \mathrm{P}<0.05$ vs. SCR cells. ATF6, activating transcription factor 6; DSPP, dentin sialophosphoprotein; GRP78, 78 kDa glucose-regulated protein; IP3r, inositol 1,4,5-trisphosphate receptor; IRE1, serine/threonine-protein kinase/endoribonuclease IRE1; MMP20, matrix metalloproteinase 20; P, puromycin; PERK, protein kinase R-like endoplasmic reticulum kinase; SCR, scrambled shRNA; SERCA2b, sarcoplasmic/endoplasmic reticulum calcium ATPase 2b; Sh, DSSP shRNA; shRNA, short hairpin RNA.
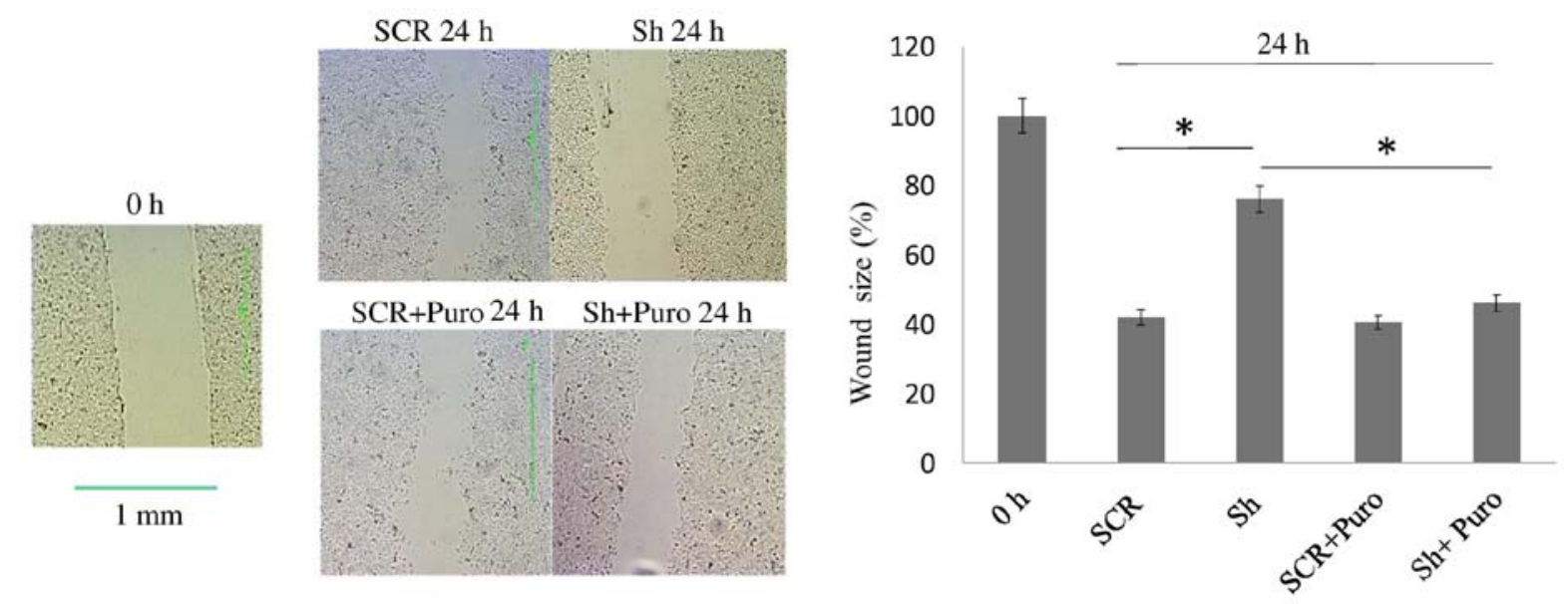

Figure 2. Effects of DSPP silencing on the migratory capacity of OSCC cells, as evaluated by a scratch wound-healing assay. DSPP silencing in puromycin-free OSCC cells resulted in a significantly delayed rate of wound closure compared with in SCR cells after $24 \mathrm{~h}$. Conversely, the rate of wound closure was comparable in puromycin-treated DSPP-silenced and SCR cells after $24 \mathrm{~h}$. Wound healing exhibited a significantly increased closure rate in puromycin-treated vs. puromycin-free DSPP-silenced cells. Data are presented as the means \pm standard error of the mean; each experiment was performed in triplicate. ${ }^{*} \mathrm{P}<0.05$. DSPP, dentin sialophosphoprotein; OSCC, oral squamous cell carcinoma; Puro, puromycin; SCR, scrambled shRNA; Sh, DSSP shRNA; shRNA, short hairpin RNA.

and SCR cells (Fig. 1B) were less pronounced compared with between the puromycin-free DSPP-silenced and SCR cells (Fig. 1A). In addition, IP3r was only downregulated in puromycin-treated cells, and PERK exhibited comparable downregulation in puromycin-free and puromycin-treated cells. Statistically significant differences were detected between GRP78 and PERK mRNA expression in DSPPsilenced cells compared with SCR cells following puromycin treatment. Notably, puromycin treatment resulted in a $38 \%$ decrease in DSPP as a result of silencing (Fig. 1B) compared with a $55 \%$ decrease in puromycin-free silenced cells (Fig. 1A). However, there was no difference on the effects of DSPP silencing on UPR activity between puromycin-treated and puromycin-free DSPP-silenced OSC2 cells.

DSPP silencing downregulates MMP20 expression and decreases the migratory potential of OSC 2 cells. To determine the effects of DSPP silencing on the migratory capacity of OSC2 cells, closure of a scratch wound in cell culture plates was measured. As shown in Fig. 2, there was a significant 


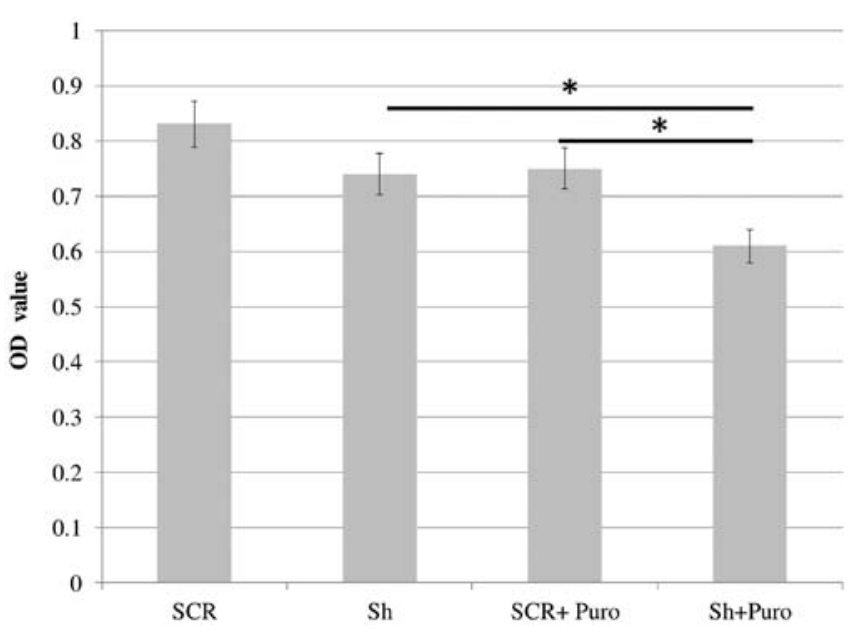

Figure 3. Oral squamous cell carcinoma cell proliferation was determined using the MTT colorimetric assay. DSPP-silenced puromycin-free cells exhibited a slight, but not significant, reduction in OD values compared with in SCR cells. In addition, puromycin-treated DSPP-silenced cells exhibited significantly lower OD values compared with in puromycin-treated SCR cells and puromycin-free DSPP-silenced cells. Data are presented as the means \pm standard error of the mean; each experiment was performed in triplicate. "P<0.05. DSPP, dentin sialophosphoprotein; OD, optical density; PUROM, puromycin; SCR, scrambled shRNA; shDSSP, DSSP shRNA; shRNA, short hairpin RNA.

delay in wound closure $(\mathrm{P}<0.05)$ in DSPP-silenced cells compared with in SCR cells after $24 \mathrm{~h}$. This finding may be associated with the significant reduction in MMP20 mRNA expression $(\mathrm{P}<0.05)$, which was observed in DSPP-silenced cells compared with in SCR cells (Fig. 1A), thus suggesting that DSPP, along with its cognate partner MMP20, may regulate the migratory capacity of OSC2 cells.

With respect to puromycin-treated OSC2 cells, wound closure rate was similar between DSPP-silenced and SCR cells after 24 h (Fig. 2). However, wound healing exhibited a significantly increased closure rate $(\mathrm{P}<0.05)$ in puromycintreated DSPP-silenced cells compared with in puromycin-free DSPP-silenced cells. Conversely, MMP20 mRNA expression levels were minimally decreased following DSPP silencing in puromycin-treated cells (Fig. 1B), and puromycin-treated DSPP-silenced cells exhibited significantly $(\mathrm{P}<0.05)$ increased MMP20 mRNA expression compared with puromycin-free DSPP-silenced cells (Fig. 1A and B). These results suggested that puromycin may antagonize the effects of DSPP silencing on MMP20 expression and may increase migration of OSC2 cells.

DSPP silencing inhibits cell proliferation and increases apoptosis of OSC2 cells. DSPP-silenced and SCR OSC2 cells were cultured in 96-well plates for $24 \mathrm{~h}$, and mitochondrial activity was determined using the MTT colorimetric assay. As shown in Fig. 3, DSPP-silenced cells exhibited a slight, but insignificant, reduction in OD values compared with in SCR cells. The rate of apoptosis of DSPP-silenced OSC2 cells was assessed by Annexin V-FITC flow cytometry; the apoptotic cell fraction was increased from $3.41 \%$ in SCR cells to $10.9 \%$ in DSPP-silenced cells (Fig. 4). Taken together, DSSP silencing reduced cell proliferation and enhanced apoptosis, thus suggesting that DSPP may exert a tumorigenic influence on OSC2 cells. With the addition of puromycin, DSPP-silenced cells exhibited significantly lower OD values
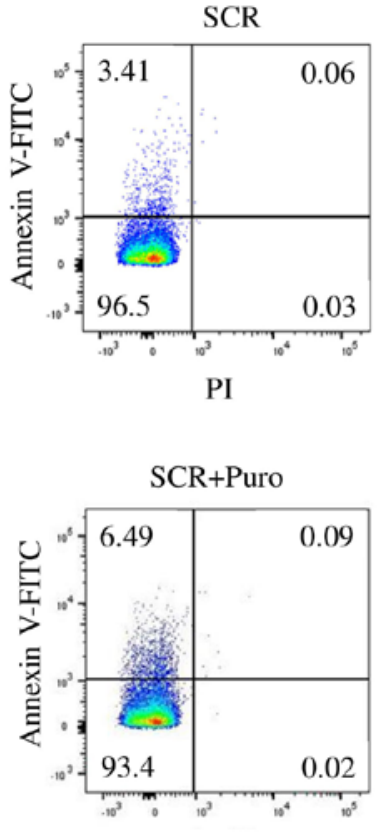

PI
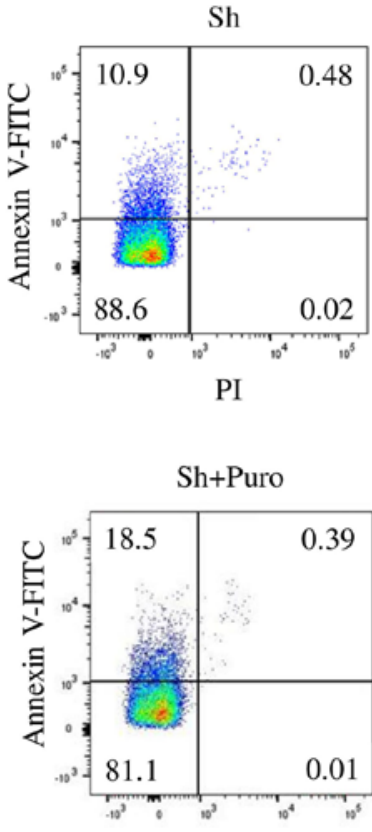

PI

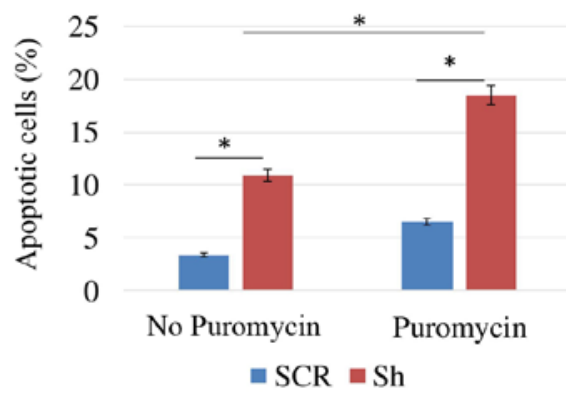

Figure 4. Rate of apoptosis in DSPP-silenced oral squamous cell carcinoma cells, as analyzed by Annexin V-FITC flow cytometry, and compared with rates in SCR cells. The apoptotic cell fraction was significantly increased from $3.38 \%$ in SCR cells to $10.9 \%$ in DSPP-silenced puromycin-free cells. The apoptotic cell fraction was significantly increased from $6.49 \%$ in puromycin-treated SCR cells to $18.5 \%$ in puromycin-treated DSPP silenced cells. Puromycin-treated DSPP-silenced cells exhibited higher apoptotic rates compared with in puromycin-free DSPP-silenced cells. Data are presented as the means \pm standard error of the mean; each experiment was performed in triplicate. ${ }^{*} \mathrm{P}<0.05$. DSPP, dentin sialophosphoprotein; FITC, fluorescein isothiocyanate; PI, propidium iodide; puro, puromycin; SCR, scrambled shRNA; Sh, DSSP shRNA; shRNA, short hairpin RNA. 


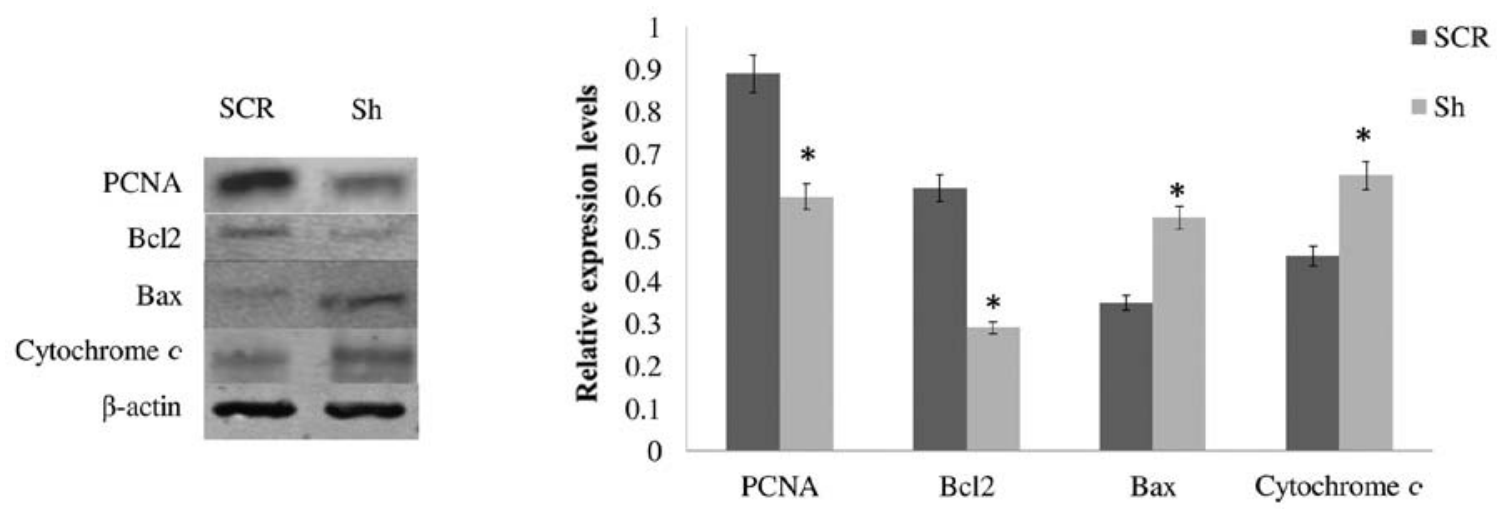

Figure 5. DSPP silencing affects the expression of cell proliferation- and apoptosis-associated proteins in oral squamous cell carcinoma cells. Western blotting detected decreases in protein expression levels of Bcl2 and PCNA, whereas Bax and cytochrome $c$ levels were increased in DSPP-silenced cells compared with in SCR cells. " $\mathrm{P}<0.05$. Data are presented as the means \pm standard error of the mean; each experiment was performed in triplicate. DSPP, dentin sialophosphoprotein; Bax, Bcl2-associated X protein; Bcl2, B-cell lymphoma 2; PCNA, proliferating cell nuclear antigen; SCR, scrambled shRNA; Sh, DSSP shRNA; shRNA, short hairpin RNA.

$(\mathrm{P}<0.05)$ compared with in SCR cells and puromycin-free DSPP-silenced cells (Fig. 3). Annexin V-FITC flow cytometry detected an increase in the apoptotic cell fraction from 3.41 to $6.49 \%$, and from 10.9 to $18.5 \%$, in response to 48 -h puromycin treatment of SCR and DSPP-silenced cells, respectively (Fig. 4). Notably, puromycin-treated DSPP-silenced cells exhibited a higher apoptotic rate than puromycin-free DSPPsilenced cells.

Effects of DSPP silencing on the expression of cell proliferation- and apoptosis-associated proteins in OSC2 cells. Since the aforementioned results indicated that puromycin-treated and puromycin-free DSPP-silenced OSC2 cells presented comparable effects with regards to the mRNA expression levels of UPR-related proteins, puromycin seems to only partially counteract, without significantly reversing, the effects of DSPP silencing on OSC2 cells. Therefore, although there were significant differences between puromycin-free and puromycin-treated cells with respect to apoptosis and proliferation, puromycin treatment, per se, did not alter the original overall effect of DSPP silencing on apoptosis and cell proliferation. Therefore, to evaluate the effects of DSPP silencing on cell proliferation and apoptosis-related proteins, western blot analysis was conducted only on whole cell lysates from puromycin-free DSPP-silenced and SCR OSC2 cells. As shown in Fig. 5, the protein expression levels of the proliferation marker PCNA and the anti-apoptotic molecule Bcl 2 were significantly decreased $(\mathrm{P}<0.05)$, whereas levels of the proapoptotic molecule, Bax, and cytochrome $c$ were increased, compared with SCR cells. These results, which are concordant with the results of cell proliferation (Fig. 3) and apoptosis (Fig. 4) analyses, indicated that alterations in DSPP expression may affect the expression and activity of cell proliferation- and apoptosis-associated molecules, thus modulating the survival of OSC 2 cells.

\section{Discussion}

The present study demonstrated that DSPP silencing may result in perturbation of ER calcium homeostasis in OSC2 cells, as evidenced by reductions in the mRNA expression levels of GRP78, SERCA2b, IRE1, PERK and ATF6. Conversely, IP3r levels remained unchanged. Furthermore, the results indicated that DSPP silencing induced a reduction in Bcl2 and PCNA protein expression, and an increase in Bax and cytochrome $c$ levels. The present data also verified the findings of a previously published study, which reported that DSPP downregulation may decrease cell proliferation and increase apoptosis of OSC2 cancer cells (33). Bcl 2 serves a cytoprotective role, exerting its prosurvival effect by binding, inhibiting and sequestrating proapoptotic proteins [Bax/Bcl2 homologous antagonist/ killer (Bak)], and by lowering ER $\mathrm{Ca}^{2+}$ stores $(34,35)$. Bcl2, expressed in ER, has also been reported to exert anti-apoptotic effects and to antagonize Bax/Bak-independent paraptotic cell death in transformed mouse kidney epithelial cells (35). Furthermore, ER stress enhances Bax translocation and insertion into the ER membrane in MEF cells (36), and Bax or Bak upregulation induces ER $\mathrm{Ca}^{2+}$ efflux and cytochrome $c$ release from the mitochondria $(37,38)$.

In general, cancer cells demonstrate increased levels of misfolded proteins and ER stress as a result of genetic mutations, stressful microenvironment, or in response to anticancer therapy $(39,40)$. Furthermore, changes in calcium homeostasis help tumor cells rearrange calcium signaling according to the activity of various cancer-associated proteins and acclimatize to a new hostile environment (41). Several components of the UPR have been revealed to be overexpressed in numerous cancer types; therefore, targeting UPR proteins has been proposed as a promising therapeutic strategy $(15,39,42)$.

The present data indicated that DSPP silencing may decrease GRP78 and SERCA2b mRNA expression, and induce oncosuppressive effects. GRP78 is a major chaperone and modulator of the UPR that demonstrates high protein-binding capacity and directly manages UPR signaling proteins IRE1, PERK and ATF6 (43). Previous studies have suggested that GRP78 promotes tumor progression $(44,45)$, and increased GRP78 expression has been correlated with reduced patient survival and shorter recurrence time in esophageal squamous cell carcinoma, prostate and breast cancer $(41,46,47)$. Other studies have indicated that GRP78 silencing reduces tumor cell invasion, cell growth and metastasis in xenograft models of gastric cancer (48). Furthermore, decreased GRP78 expression reduces multidrug resistance (49). 
SERCA regulates cellular $\mathrm{Ca}^{2+}$ homeostasis via its pump activity, which channels $\mathrm{Ca}^{2+}$ into the ER, thereby maintaining the requisite ionic balance between ER and cytosolic calcium (50). In addition to this role, SERCA is involved in survival pathways, including the Notch1 signaling pathway (51). SERCA inhibition has been reported to cause $\mathrm{ER} \mathrm{Ca}^{2+}$ depletion, while increasing cytosolic and mitochondrial $\mathrm{Ca}^{2+}$ concentration. SERCA pump inhibitors, such as thapsigargin, which are capable of interfering with calcium homeostasis, have been revealed to exert cytotoxic effects on cancer cells in vitro $(15,52)$. Specifically, persistent $\mathrm{ER} \mathrm{Ca}^{2+}$ depletion may activate the ER stress response and intrinsic apoptotic pathways involving caspase 12 , whereas high cytosolic calcium levels may trigger extrinsic proapoptotic and anti-apoptotic signaling of $\mathrm{Bcl} 2$ family members with subsequent release of mitochondrial cytochrome $c$ (15). It has also been suggested that Bcl2 directly interacts with SERCA resulting in alterations in calcium pumps and calcium levels required for initiating apoptosis (53).

The present results also revealed that DSPP silencing induced downregulation of the three major UPR-sensors (PERK, ATF6 and IRE1), whose activities have been associated with tumorigenic effects. Cancer cells appear more resistant to environmental stress due to alterations in the UPR protein activity (18). PERK and its target molecule eukaryotic initiation factor $2 \alpha$ have been associated with increased tumor growth and survival under hypoxic conditions (28). Conversely, PERK inhibition decreases tumor growth in vitro and in vivo (54), impedes cell cycle progression and reduces metastatic propensity in mammary cancer in mice xenografts (55). Similarly, whereas IRE1 contributes to resistance against ER stress-mediated cell death through its effects on Bax/Bak activity, its inhibition results in oligomerization of Bax and Bak and an increase in ER membrane permeabilization (56). High levels of ATF6 protein have also been detected in several human solid tumors and it has been suggested to induce proliferation and survival under nutrient-deficient conditions (30). Previous reports suggested that ATF6 enhances X-box binding protein 1 expression, and both proteins increase GRP78 activity in liver cancer (57). Furthermore, ATF6 activation results in radiation-induced upregulation of GRP78 and Notch1, whereas ATF6 knockdown promotes radiation-induced cell death in glioblastoma (58).

The results of a scratch wound-healing assay indicated that DSPP silencing in OSC2 cells reduced migration compared with the control cells. This effect may be attributable to a decrease in MMP20 levels upon DSPP silencing. Recently, MMP20 was identified as the cognate MMP partner of DSPP (13). Furthermore, it has been reported that the DSPP cleaved product, DSP, interacts with MMP20 through its $\mathrm{N}$-terminal domain. It has been suggested that this interaction may bridge MMP20 to cell surface receptors, thus triggering signaling pathways associated with proliferation, migration, invasion and metastasis (13). Other investigators have reported that GRP78 downregulation, which results in decreased MMP2 and MMP9 expression, significantly reduces the metastatic potential of esophageal SCC cells (59). Furthermore, GRP78 knockdown downregulates TIMP metallopeptidase inhibitor (TIMP)1, TIMP2, MMP14, MMP2 and MMP9 expression in human pancreatic adenocarcinoma cells (60). It therefore may be hypothesized that the decreased migratory ability of DSPP- silenced cells is due to the consequent reduction in MMP20 and GRP78 mRNA expression. As has been revealed for other MMPs, this finding indicated that GRP78 may be a potential regulator of MMP20.

The present study analyzed the effects of puromycin treatment on DSPP-silenced oral cancer cells, and demonstrated that treatment of OSC2 cells with puromycin partially counteracted the effects of DSSP silencing on GRP78, SERCA, IRE1 and ATF6 expression, without reversing the overall effects of DSPP silencing on OSC2 cells. Puromycin-treated DSPP-silenced OSC2 cells exhibited less pronounced reductions in GRP78, SERCA, IRE1 and ATF6 expression compared with DSPP-silenced cells without puromycin treatment. Furthermore, puromycin-treated DSPP-silenced cells exhibited further reductions in cell proliferation and increased apoptosis compared with DSPP-silenced cells without puromycin treatment. A plausible explanation for this result is that puromycin may trigger the UPR, disrupting protein synthesis and the metabolic activity of OSC 2 cells. Both puromycintreated and untreated DSPP-silenced cells exhibited low SERCA2b mRNA expression.

As a translocon opener, puromycin treatment of cells may result in increased ER calcium release and higher rates of apoptosis. For example, Oguma et al reported that puromycin treatment induces GRP78 expression, resulting in caspase 12-mediated cell death in cluster of differentiation (CD) $4^{+} \mathrm{CD} 8^{+}$thymic lymphoma cells and in normal thymocytes (61). Johnson et al also revealed that malignant glioma cells treated with a low dose of puromycin exhibit increased GRP78 protein and mRNA levels, and enhanced activation of caspase 4. Caspase 4 activation has been suggested to result from the direct effect of calcium leakage through open translocons, leading to $\sim 93 \%$ reduction of cell viability (62). Similarly, it has been reported that puromycin treatment of human prostate adenocarcinoma cells increases GRP78 protein expression, enhances ER calcium release via translocons, and induces apoptosis (63).

In the context of the present study, the aforementioned effects of puromycin on DSPP-silenced cells may also account for the less pronounced reduction in MMP20 mRNA. Alternatively, the less pronounced downregulation of MMP20 caused by puromycin treatment in DSPP-silenced cells may be due to less pronounced reductions in GRP78 levels in puromycin-treated cells. The composite effect may be increased cell migration, as indicated by the scratch wound-healing assay. Previous studies reported that puromycin treatment induces GRP78 expression and leads to caspase activation in several malignancies $(62,63)$; this has been suggested to be a direct effect of enhanced ER calcium release via translocon $(62,63)$. Therefore, in the present study, puromycin was used as a vehicle to cause alterations in the expression of GRP78 and UPR-related proteins, as well as in ER calcium homeostasis. Since the present study focused on the tumorigenic role of DSPP and its potential correlation with alterations in the UPR and ER stress, puromycin treatment revealed how the observed changes may affect DSPP tumorigenic activity in OSC2 cells. To the best of our knowledge, the present study is the first to provide an insight into the potential associations between DSPP and GRP78 functional roles, and the modulation of ER stress in OSCC. 
DSPP is an evolutionary younger member of the acidic secretory calcium-binding phosphoprotein gene family (7). The two major DSPP cleaved functional units, DSP and DPP, are highly acidic, with human DPP presenting a very low isoelectric point of 2.84 and high calcium affinity $(7,8)$. It is therefore conceivable that DSPP serves as a buffer protein in ER calcium homeostasis with a similar functional role to other calcium-dependent proteins: Calnexin, calreticulin and GRP78 (64). Furthermore, the DSPP cysteine residue may function similarly to its GRP78 counterpart, which has been suggested to promote cell survival under ER stress (27), and by doing so may contribute to cell stability during ER stress. Therefore, DSPP silencing may lead to a sudden alteration in the ER calcium balance, provoking collapse of the UPR as a result of the calcium-demanding tumor microenvironment.

In conclusion, the present study provided evidence to suggest that DSPP is involved in ER stress mechanisms. Downregulation of DSPP in OSCC cells resulted in changes in major ER stress-associated proteins (GRP78, SERCA2b and UPR sensor proteins), thus leading to collapse of the UPR system and function. These results also validated previously published reports, indicating that DSPP silencing resulted in decreased cell proliferation, migration, invasion and increased apoptosis. Further in-depth studies are required to determine the nature of the interaction/relationship between DSPP and major UPR regulators, such as GRP78, in OSCC. Increased understanding may advance the understanding of the ability of cancer cells to survive under stressful conditions, and to elucidate potential therapeutic strategies.

\section{Acknowledgements}

Not applicable.

\section{Funding}

The present study was supported by faculty startup research funding (to KUEO) from the University of Texas Health Science Center at Houston (Houston, TX, USA).

\section{Availability of data and materials}

The datasets used and/or analyzed during the present study are available from the corresponding author on reasonable request.

\section{Authors' contributions}

NGN and KUEO made substantial contributions to the conception and design of the study, reviewed data, reviewed/ edited draft manuscripts, and reviewed/edited the final draft of the manuscript. IG and JA carried out experiments related to the study, acquired, analyzed and interptreted data, and provided the initial draft of the manuscript. All authors gave their approval of the final draft of the manuscript, and agree to be accountable for all aspects of the study related to accuracy or integrity of all parts of the study.

\section{Ethics approval and consent to participate}

Not applicable.

\section{Patient consent for publication}

Not applicable.

\section{Competing interests}

The authors declare that they have no competing interests.

\section{References}

1. Bellahcène A, Castronovo V, Ogbureke KU, Fisher LW and Fedarko NS: Small integrin-binding ligand N-linked glycoproteins (SIBLINGs): Multifunctional proteins in cancer. Nat Rev Cancer 8: 212-226, 2008

2. Ogbureke KU and Fisher LW: Expression of SIBLINGs and their partner MMPs in salivary glands. J Dent Res 83: 664-670, 2004.

3. Ogbureke KU and Fisher LW: SIBLING expression patterns in duct epithelia reflect the degree of metabolic activity. J Histochem Cytochem 55: 403-409, 2007.

4. Koli K, Saxena G and Ogbureke KU: Expression of matrix metalloproteinase (MMP)-20 and potential interaction with dentin sialophosphoprotein (DSPP) in human major salivary glands. J Histochem Cytochem 63: 524-533, 2015.

5. Yamakoshi Y, Hu JC, Iwata T, Kobayashi K, Fukae M and Simmer JP: Dentin sialophosphoprotein is processed by MMP-2 and MMP-20 in vitro and in vivo. J Biol Chem 281: 38235-38243, 2006.

6. Suzuki S, Sreenath T, Haruyama N, Honeycutt C, Terse A, Cho A, Kohler T, Müller R, Goldberg M and Kulkarni AB: Dentin sialoprotein and dentin phosphoprotein have distinct roles in dentin mineralization. Matrix Biol 28: 221-229, 2009.

7. Yang J, Kawasaki K, Lee M, Reid BM, Nunez SM, Choi M, Seymen F, Koruyucu M, Kasimoglu Y, Estrella-Yuson N, et al: The dentin phosphoprotein repeat region and inherited defects of dentin. Mol Genet Genomic Med 4: 28-38, 2015.

8. Alvares K, Stern PH and Veis A: Dentin phosphoprotein binds annexin 2 and is involved in calcium transport in rat kidney ureteric bud cells. J Biol Chem 288: 13036-13045, 2013.

9. Fisher LW, Jain A, Tayback M and Fedarko NS: Small integrin binding ligand $\mathrm{N}$-linked glycoprotein gene family expression in different cancers. Clin Cancer Res 10: 8501-8511, 2004.

10. Jain A, McKnight DA, Fisher LW, Humphreys EB, Mangold LA, Partin AW and Fedarko NS: Small integrin-binding proteins as serum markers for prostate cancer detection. Clin Cancer Res 15: 5199-5207, 2009.

11. Anunobi CC, Koli K, Saxena G, Banjo AA and Ogbureke KU: Expression of the SIBLINGs and their MMP partners in human benign and malignant prostate neoplasms. Oncotarget 7: 48038-48049, 2016

12. Ogbureke KU, Nikitakis NG, Warburton G, Ord RA, Sauk JJ, Waller JL and Fisher LW: Up-regulation of SIBLING proteins and correlation with cognate MMP expression in oral cancer. Oral Oncol 43: 920-932, 2007.

13. Saxena G, Koli K, de la Garza J and Ogbureke KU: Matrix metalloproteinase 20-dentin sialophosphoprotein interaction in oral cancer. J Dent Res 94: 584-593, 2015.

14. Rao RV, Hermel E, Castro-Obregon S, del Rio G, Ellerby LM, Ellerby HM and Bredesen DE: Coupling endoplasmic reticulum stress to the cell death program. Mechanism of caspase activation. J Biol Chem 276: 33869-33874, 2001.

15. Denmeade SR and Isaacs JT: The SERCA pump as a therapeutic target: Making a 'smart bomb' for prostate cancer. Cancer Biol Ther 4: 14-22, 2005.

16. Mekahli D, Bultynck G, Parys JB, De Smedt H and Missiaen L: Endoplasmic-reticulum calcium depletion and disease. Cold Spring Harb Perspect Biol 3: 3, 2011.

17. Raturi A, Ortiz-Sandoval C and Simmen T: Redox dependence of endoplasmic reticulum (ER) $\mathrm{Ca}^{2+}$ signaling. Histol Histopathol 29: 543-552, 2014.

18. Giampietri C, Petrungaro S, Conti S, Facchiano A, Filippini A and Ziparo E: Cancer microenvironment and endoplasmic reticulum stress response. Mediators Inflamm 2015: 417281, 2015.

19. Wang M and Kaufman RJ: The impact of the endoplasmic reticulum protein-folding environment on cancer development. Nat Rev Cancer 14: 581-597, 2014. 
20. Lemus L and Goder V: Regulation of endoplasmic reticulumassociated protein degradation (ERAD) by ubiquitin. Cells 3 : 824-847, 2014.

21. Yadav RK, Chae SW, Kim HR and Chae HJ: Endoplasmic reticulum stress and cancer. J Cancer Prev 19: 75-88, 2014.

22. Bertolotti A, Zhang Y, Hendershot LM, Harding HP and Ron D: Dynamic interaction of BiP and ER stress transducers in the unfolded-protein response. Nat Cell Biol 2: 326-332, 2000

23. Brown JM and Giaccia AJ: The unique physiology of solid tumors: Opportunities (and problems) for cancer therapy. Cancer Res 58: 1408-1416, 1998.

24. He B: Viruses, endoplasmic reticulum stress, and interferon responses. Cell Death Differ 13: 393-403, 2006.

25. Martinon F: Targeting endoplasmic reticulum signaling pathways in cancer. Acta Oncol 51: 822-830, 2012.

26. Moenner M, Pluquet O, Bouchecareilh $M$ and Chevet E Integrated endoplasmic reticulum stress responses in cancer. Cancer Res 67: 10631-10634, 2007.

27. Wang $\mathrm{J}$ and Sevier CS: Formation and reversibility of BiP protein cysteine oxidation facilitate cell survival during and post oxidative stress. J Biol Chem 291: 7541-7557, 2016.

28. Koumenis C: ER stress, hypoxia tolerance and tumor progression. Curr Mol Med 6: 55-69, 2006.

29. Fu Y, Li J and Lee AS: GRP78/BiP inhibits endoplasmic reticulum BIK and protects human breast cancer cells against estrogen starvation-induced apoptosis. Cancer Res 67: 3734-3740, 2007

30. Ye J, Kumanova M, Hart LS, Sloane K, Zhang H, De Panis DN, Bobrovnikova-Marjon E, Diehl JA, Ron D and Koumenis C: The GCN2-ATF4 pathway is critical for tumour cell survival and proliferation in response to nutrient deprivation. EMBO J 29: 2082-2096, 2010.

31. Thorpe JA and Schwarze SR: IRE1alpha controls cyclin A1 expression and promotes cell proliferation through XBP-1. Cell Stress Chaperones 15: 497-508, 2010.

32. Livak KJ and Schmittgen TD: Analysis of relative gene expression data using real-time quantitative PCR and the 2(-Delta Delta C(T)) Method. Methods 25: 402-408, 2001

33. Joshi R, Tawfik A, Edeh N, McCloud V, Looney S, Lewis J, Hsu S and Ogbureke KU: Dentin sialophosphoprotein (DSPP) gene-silencing inhibits key tumorigenic activities in human ora cancer cell line, OSC2. PLoS One 5: e13974, 2010.

34. Brunelle JK and Letai A: Control of mitochondrial apoptosis by the Bcl-2 family. J Cell Sci 122: 437-441, 2009.

35. Heath-Engel HM, Wang B and Shore GC: Bcl2 at the endoplasmic reticulum protects against a $\mathrm{Bax} / \mathrm{Bak}$-independent paraptosislike cell death pathway initiated via p20Bap31. Biochim Biophys Acta 1823: 335-347, 2012

36. Wang $\mathrm{X}$, Olberding $\mathrm{KE}$, White $\mathrm{C}$ and $\mathrm{Li} \mathrm{C}$ : $\mathrm{Bcl}-2$ proteins regulate ER membrane permeability to luminal proteins during ER stress-induced apoptosis. Cell Death Differ 18: 38-47, 2011.

37. Nutt LK, Pataer A, Pahler J, Fang B, Roth J, McConkey DJ and Swisher SG: Bax and Bak promote apoptosis by modulating endoplasmic reticular and mitochondrial $\mathrm{Ca}^{2+}$ stores. J Biol Chem 277: 9219-9225, 2002.

38. Scorrano L, Oakes SA, Opferman JT, Cheng EH, Sorcinelli MD, Pozzan T and Korsmeyer SJ: BAX and BAK regulation of endoplasmic reticulum $\mathrm{Ca}^{2+}$ : A control point for apoptosis. Science 300: 135-139, 2003

39. Nagelkerke A, Bussink J, Sweep FC and Span PN: The unfolded protein response as a target for cancer therapy. Biochim Biophys Acta 1846: 277-284, 2014

40. Corazzari M, Gagliardi M, Fimia GM and Piacentini $M$ : Endoplasmic reticulum stress, unfolded protein response, and cancer cell fate. Front Oncol 7: 78, 2017.

41. Marchi S and Pinton P: Alterations of calcium homeostasis in cancer cells. Curr Opin Pharmacol 29: 1-6, 2016

42. Lee E, Nichols P, Spicer D, Groshen S, Yu MC and Lee AS: GRP78 as a novel predictor of responsiveness to chemotherapy in breast cancer. Cancer Res 66: 7849-7853, 2006

43. de Ridder G, Ray R, Misra UK and Pizzo SV: Modulation of the unfolded protein response by GRP78 in prostate cancer. Methods Enzymol 489: 245-257, 2011

44. Dong D, Ni M, Li J, Xiong S, Ye W, Virrey JJ, Mao C, Ye R, Wang M,Pen L, et al: Critical role of the stress chaperone GRP78/ $\mathrm{BiP}$ in tumor proliferation, survival, and tumor angiogenesis in transgene-induced mammary tumor development. Cancer Res 68: 498-505, 2008
45. Fu Y, Wey S, Wang M, Ye R, Liao CP, Roy-Burman P and Lee AS Pten null prostate tumorigenesis and AKT activation are blocked by targeted knockout of ER chaperone GRP78/BiP in prostate epithelium. Proc Natl Acad Sci USA 105: 19444-19449, 2008.

46. Daneshmand S, Quek ML, Lin E, Lee C, Cote RJ, Hawes D, Cai J, Groshen S, Lieskovsky G, Skinner DG, et al: Glucose-regulated protein GRP78 is up-regulated in prostate cancer and correlates with recurrence and survival. Hum Pathol 38: 1547-1552, 2007.

47. Ren P, Chen C, Yue J, Zhang J and Yu Z: High expression of glucose-regulated protein 78 (GRP78) is associated with metastasis and poor prognosis in patients with esophageal squamous cell carcinoma. OncoTargets Ther 10: 617-625, 2017.

48. Zhang J, Jiang Y, Jia Z, Li Q, Gong W, Wang L, Wei D, Yao J, Fang S and Xie K: Association of elevated GRP78 expression with increased lymph node metastasis and poor prognosis in patients with gastric cancer. Clin Exp Metastasis 23: 401-410, 2006.

49. Kang J, Zhao G, Lin T, Tang S, Xu G, Hu S, Bi Q, Guo C, Sun L, Han S, et al: A peptide derived from phage display library exhibits anti-tumor activity by targeting GRP78 in gastric cancer multidrug resistance cells. Cancer Lett 339: 247-259, 2013.

50. Toyoshima C, Nomura H and Sugita Y: Crystal structures of $\mathrm{Ca}^{2+}$-ATPase in various physiological states. Ann N Y Acad Sci 986: $1-8,2003$

51. Roti G, Carlton A, Ross KN, Markstein M, Pajcini K, Su AH, Perrimon N, Pear WS, Kung AL, Blacklow SC, et al: Complementary genomic screens identify SERCA as a therapeutic target in NOTCH1 mutated cancer. Cancer Cell 23: 390-405, 2013

52. Casemore D and Xing C: SERCA as a target for cancer therapies. Integr Cancer Sci Ther 2: 100-103, 2015.

53. Dremina ES, Sharov VS, Kumar K, Zaidi A, Michaelis EK and Schöneich C: Anti-apoptotic protein Bcl-2 interacts with and destabilizes the sarcoplasmic/endoplasmic reticulum $\mathrm{Ca}^{2+}$-ATPase (SERCA). Biochem J 383: 361-370, 2004.

54. Axten JM, Medina JR, Feng Y, Shu A, Romeril SP, Grant SW, Li WH, Heerding DA, Minthorn E, Mencken T, et al: Discovery of 7-methyl-5-(1-\{[3-(trifluoromethyl)phenyl]acetyl $\}$ 2,3-dihydro-1H-indol-5-yl)-7H-pyrrolo[2,3-d]pyrimidin-4-amine (GSK2606414), a potent and selective first-in-class inhibitor of protein kinase R (PKR)-like endoplasmic reticulum kinase (PERK). J Med Chem 55: 7193-7207, 2012.

55. Bobrovnikova-Marjon E, Grigoriadou C, Pytel D, Zhang F, Ye J, Koumenis C, Cavener D and Diehl JA: PERK promotes cancer cell proliferation and tumor growth by limiting oxidative DNA damage. Oncogene 29: 3881-3895, 2010

56. Kanekura K, Ma X, Murphy JT, Zhu LJ, Diwan A and Urano F: IRE1 prevents endoplasmic reticulum membrane permeabilization and cell death under pathological conditions. Sci Signal 8: ra62, 2015

57. Shuda M, Kondoh N, Imazeki N, Tanaka K, Okada T, Mori K, Hada A, Arai M, Wakatsuki T, Matsubara O, et al: Activation of the ATF6, XBP1 and grp78 genes in human hepatocellular carcinoma: A possible involvement of the ER stress pathway in hepatocarcinogenesis. J Hepatol 38: 605-614, 2003

58. Dadey DY, Kapoor V, Khudanyan A, Urano F, Kim AH, Thotala D and Hallahan DE: The ATF6 pathway of the ER stress response contributes to enhanced viability in glioblastoma. Oncotarget 7: 2080-2092, 2016.

59. Zhao G, Kang J, Jiao K, Xu G, Yang L, Tang S, Zhang H, Wang Y, Nie Y, Wu K, et al: High expression of GRP78 promotes invasion and metastases in patients with esophageal squamous cell carcinoma. Dig Dis Sci 60: 2690-2699, 2015.

60. Yuan XP, Dong M, Li X and Zhou JP: GRP78 promotes the invasion of pancreatic cancer cells by FAK and JNK. Mol Cell Biochem 398: 55-62, 2015.

61. Oguma T, Ono T, Kajiwara T, Sato M, Miyahira Y, Arino H, Yoshihara Y and Tadakuma T: CD4(+)CD8(+) thymocytes are induced to cell death by a small dose of puromycin via ER stress. Cell Immunol 260: 21-27, 2009.

62. Johnson GG, White MC, Wu JH, Vallejo M and Grimaldi M The deadly connection between endoplasmic reticulum, $\mathrm{Ca}^{2+}$ protein synthesis, and the endoplasmic reticulum stress response in malignant glioma cells. Neuro-oncol 16: 1086-1099, 2014.

63. Hammadi M, Oulidi A, Gackière F, Katsogiannou M, Slomianny C, Roudbaraki M, Dewailly E, Delcourt P, Lepage G, Lotteau S, et al: Modulation of ER stress and apoptosis by endoplasmic reticulum calcium leak via translocon during unfolded protein response: Involvement of GRP78. FASEB J 27: 1600-1609, 2013.

64. Michalak M, Milner RE, Burns K and Opas M: Calreticulin. Biochem J 285: 681-692, 1992. 